\title{
Optimal Environmental and Economic Load Dispatch of Power Systems Based on the Pareto Front of Particle Swarm Optimization
}

\author{
Chaofan Zong, Zhigang Lu and Jie He \\ The Electrical Engineering College, Guizhou University, Guiyang, 550000, China
}

Keywords: environmental and economic load dispatch, pareto front, improved PSO, multiobjective

\begin{abstract}
Currently, most of multi-objective problems in power systems are changed into a single objective problem by weighting. This paper adopts Pareto front to solve the multi-objective problems in power systems. In order to solve this problemeffectively, we present the particle swarm optimization(PSO) algorithm. In addition, we improve PSO with the random black hole strategy, The dynamic update of inertia weight and learning factor, leading particles selection, small probability random mutation. The proposed algorithm can search the Pareto front of the multiobjective functions quickly, and the solutions have the diversity and uniformity. Finally, The algorithm is applied to solve the environmental and economic load dispatch problem of power systems. Considering the environmental impact of $\mathrm{CO}_{2}$ emissions and the minimum fuel consumption costs of generators, we get the optimal dispatch of generators output depending on the every period load demands.
\end{abstract}

\section{Introduction}

The economic load dispatch (ELD) is the optimal load dispatch of the power systems or power plants to minimize the generation costs[1]. Besides, it should meet the load balance and system operation constraints. With the economic development and the realization of sustainable development strategy goals, on the one hand, the electricity demands is increased and energy resources are consumed quickly; on the other hand, people hope to conserve resources and protect the environment. Therefore, the power system optimization problems are not only considered the fuel costs of generators, but also take the environmental impact of harmful gases emissions.

\section{Mathematical model of the environmental and economic load dispatch}

\subsection{Fuel costs of generators}

Fuel costs of the generator are the fuel consumption costs during the process of generation. Fuel costs formula of generators are represented as Eq.(1).

$$
C\left(P_{G i}(t)\right)=a_{i} P_{G i}^{2}(t)+b_{i} P_{G i}(t)+c_{i}, \quad i=1,2,3, \ldots, \mathrm{N} .
$$

Among them, $C\left(P_{G i}(t)\right)$ are the fuel consumption costs of the i-th generator in period t (unit: $\$$ / h); $P_{G i}(t)$ is the output active power of the $\mathrm{i}$-th generator; $\mathrm{N}$ is the number of generators in the power system; $a_{i}, b_{i}, c_{i}$ is the fuel consumption curve parameters of the i-th generator.

Thus, the total fuel costs of the power system in a scheduling period $\mathrm{T}$ can be written as the Eq.(2).

$$
f_{\text {fuel }}=\sum_{t=1}^{T} \sum_{i=1}^{N} C\left(P_{G i}(t)\right) .
$$

Among them, $i=1,2,3, \ldots, \mathrm{N} ; \mathrm{t}=1,2,3, \ldots, \mathrm{T}$. $\mathrm{T}$ is the scheduling period.

\section{2 environmental damage index of gas emissions}

Environmental damage index of gas emissions is the harmful gas emissions masses produced by generators fuel consumption multiplied by the unit punishment ratio of the corresponding gas. The major harmful gases produced by power generation of generators are carbon dioxide $\left(\mathrm{CO}_{2}\right)$, sulfur 
dioxide $\left(\mathrm{SO}_{2}\right)$ and nitrogen oxides $\left(\mathrm{NO}_{x}\right)$. The environmental damage index is calculated by the gas emissions masses and a standard of punishment ratio[2]. Three harmful gas emissions per unit time $(\mathrm{t} / \mathrm{h})$ are as follows:

$$
\begin{gathered}
E_{\mathrm{CO}_{2} i}\left(P_{G i}(t)\right)=\alpha_{C i} P_{G i}^{2}(t)+\beta_{C i} P_{G i}(t)+\gamma_{C i}+k_{C i} \exp \left(\lambda_{C i} P_{G i}(t)\right) ; \\
E_{S_{2} i}\left(P_{G i}(t)\right)=\alpha_{S i} P_{G i}^{2}(t)+\beta_{S i} P_{G i}(t)+\gamma_{S i}+k_{S i} \exp \left(\lambda_{S i} P_{G i}(t)\right) ; \\
E_{N O_{x} i}\left(P_{G i}(t)\right)=\alpha_{N i} P_{G i}^{2}(t)+\beta_{N i} P_{G i}(t)+\gamma_{N i}+k_{N i} \exp \left(\lambda_{N i} P_{G i}(t)\right) .
\end{gathered}
$$

Among them, $E_{\mathrm{CO}_{2} i}\left(P_{G i}(t)\right), E_{\mathrm{SO}_{2} i}\left(P_{G i}(t)\right), E_{\mathrm{NO}_{x} i}\left(P_{G i}(t)\right)$ are the $\mathrm{CO}_{2}, \mathrm{SO}_{2}$ and $\mathrm{NO}_{x}$ emissions of the $\mathrm{i}$-th generator generation in period t respectively; $\alpha_{C i}, \beta_{C i}, \gamma_{C i}, k_{C i}, \lambda_{C i}$ are the $C O_{2}$ emission parameters of the i-th generator; $\alpha_{S i}, \beta_{S i}, \gamma_{S i}, k_{S i}, \lambda_{S i}$ are the $S O_{2}$ emission parameters of the i-th generator; $\alpha_{N i}, \beta_{N i}, \gamma_{N i}, k_{N i}, \lambda_{N i}$ are the $N O_{x}$ emission parameters of the i-th generator.

Therefore, in the scheduling period $\mathrm{T}$, the gas emissions environmental damage index of the power system in a scheduling period T can be given as the Eq.(6).

$$
f_{\text {emission }}=\sum_{i=1}^{T} \sum_{i=1}^{N}\left(\omega_{C} E_{\mathrm{CO}_{2} i}\left(P_{G i}(t)\right)+\omega_{S} E_{\mathrm{SO}_{2} i}\left(P_{G i}(t)\right)+\omega_{N} E_{\mathrm{NO}_{x^{i}} i}\left(P_{G i}(t)\right)\right. \text {. }
$$

Among them, $\omega_{C}, \omega_{S}, \omega_{N}$ are the sum of environmental values and punishment ratios, which are the unit emission parameters of $\mathrm{CO}_{2}, \mathrm{SO}_{2}$ and $\mathrm{NO}_{x}$ respectively.

\subsection{Environmental and economic load dispatch optimization model}

\subsubsection{The objective function}

The true meaning of Multi-objective is based on the pareto front. It is not simply a weighted sum of the multi-objective functions, but the optimal set of feasible solutions which is visually showed by pareto front graphs. The pareto front graphs can show the relationship between each object. Hence, the objective functions are Eq. (2) and Eq.(6).

\subsubsection{Constraints}

I. The active power constraints of generators:

$P_{G i \min } \leq P_{G i}(t) \leq P_{G i \max }$.

Among them, $P_{G i \min }, P_{G i \max }$ are the minimum and the maximun output active power of the i-th generator.

II. The active power balance constraints of power systems:

$$
\sum_{i=1}^{N} P_{G i}(t)=P_{\text {loss }}(t)+P_{L}(t)
$$

Among them, $P_{\text {loss }}, P_{L}$ are the grid loss and load of the power system.

III. rotating reserve capacity of generators constraints:

$$
\sum_{i=1}^{N} P_{G i}^{\max }(t) \geq P_{L}(t)+S(t), \mathrm{t}=1,2,3, \ldots, \mathrm{T} .
$$

Among them, $\mathrm{S}(\mathrm{t})$ is the required reserve capacity of the power system.

\section{Improved multi-objective particle swarm optimization algorithm}

particle swarm optimization(PSO) is a kind of self-improvement, group collaboration and swarm competition algorithm[3]. It is simple in theory, less set parameters, and easy to implement, iterating fast, good in global searching. In this paper,we improve particle swarm optimization algorithm.

\subsection{Random black hole strategy}

In order to accelerate the PSO iteration speed and strengthen the global searching ability, the speed and position update formula adopt a random black hole strategy. we improve inertia weight, and use the dynamic learning factor.Choose the global optimal particle as a black hole[4]. Within 
the range of radius $\mathrm{R}$, the particles have a certain probability drawing in the black hole. Beyond the radius $R$, the particles update the position and velocity in the original way. In addition, the particles which are in the black hole have a probability escaping from the black hole. Particles not only extend the search range, but also improve the ability of searching the global optimum and greatly accelerate the searching speed.

The Fundamental of IRBHPSO algorithm is shown in the following figure.

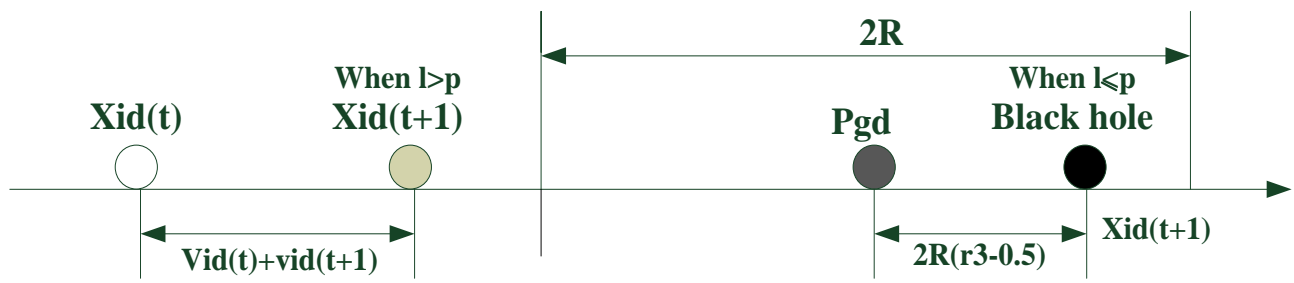

Fig.1: illustration of the IRBH Fundamental

In the each generation and dimension, we randomly propose a particle close to the current optimal particle as a black hole.Set a threshold $\mathrm{p}$ which means the magnetism of the black hole, $\mathrm{p}$ $\in[0,1]$. In each dimension of every particle, set a value $l$ which is a random number, $l \in[0,1]$. If $l<p$, let the black hole capture the particle. Otherwise, update the position and velocity in the PSO way.

Its position and velocity updating formulas are shown below. And the updating formulas are improved.

$$
\begin{gathered}
\text { When } l_{i d}^{k} \geq p, \quad v_{i d}^{k+1}=\omega v_{i d}^{k}+c\left[r 1\left(x_{p i d}^{k}-x_{i d}^{k}\right)+r 2\left(x_{g i d}^{k}-x_{i d}^{k}\right)\right], \\
x_{i d}^{k+1}=x_{i d}^{k}+v_{i d}^{k+1} .
\end{gathered}
$$

$$
\text { When } l_{i d}^{k}<p, x_{i d}^{k+1}=x_{g d}^{k}+2 R(r 3-0.5) \text {. }
$$

Among them, r1, r2, r3 are the random numbers which belong to [0,1]. $l_{i d}^{k}$ is the probability of i_th particle on the d-th dimension when it is the k-th iteration. It means the probability that particles escape from the black hole. $\mathrm{R}$ is the radius of the black hole.

\subsection{The dynamic update of inertia weight and learning factor}

Inertia weight $\omega$ and learning factor c update themselves dynamically. The following formulas Eq.(13) and Eq. (14) are given.

$$
\begin{gathered}
\omega=\omega_{0}+r 4\left(1-\omega_{0}\right) . \\
c=c_{0}+\frac{t}{M t} .
\end{gathered}
$$

Among them, $\mathrm{r} 4$ is a random number, $\mathrm{r} 4 \in[0,1]$; $\omega 0$ is a constant, $\omega 0 \in[0,0.5]$; $\mathrm{c} 0$ is a constant, $\mathrm{c} 0 \in[0.5,1]$; $\mathrm{t}$ is the number of iterations; $\mathrm{Mt}$ is the maximum number of iterations. Large inertia weight enhances global searching capability, and small inertia weight strengthen the capacity of local searching. The update method of learning factor improve the capacity of the global searching in the later searching period[5].

\subsection{NSGA-II non-dominated sorting}

This paper makes use of NSGA-II non-dominated sorting. The idea of NSGA-II non-dominated sorting has become the the mainstream method of solving the multi-objective optimization problem to get the pareto optimal solution set. The specific operation is shown in Fig.2. 


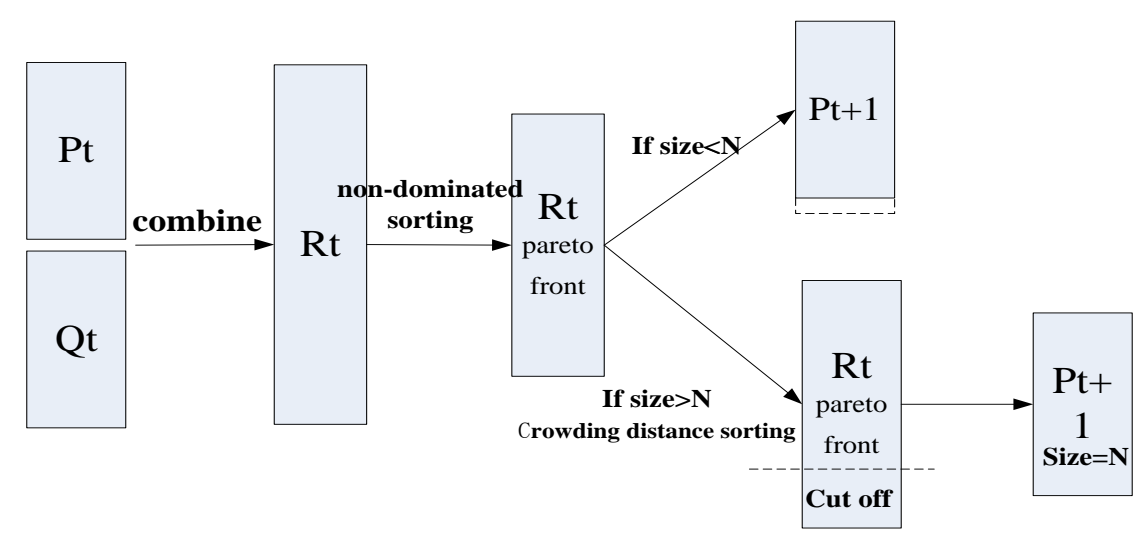

Fig.2: The specific operation of non-dominated sorting

If the population of particle swarm is $\mathrm{N}$, to be the diversity of the population, first of all, we combinate the dominated solution set Pt and the offspring solution set Qt. It forms a new particle swarm Rt (population is $2 \mathrm{~N}$ ). Calculate the fitness values of the new particle swarm $\mathrm{R}$ and do its non-dominated sorting. Then, we get the Pareto optimal front solution set of Rt. If the number of Rt's Pareto optimal front solutions is equal or less than N, we elect Rt's Pareto optimal front solutions as the next generation particle swarm $P_{t+1}$. In addition, if the number of Rt's Pareto optimal front solutions is greater than $\mathrm{N}$, we do the crowding distance sorting of its optimal front solutions. In order to ensure the uniformity of Pareto optimal front solutions distribution, we delete the small crowding distance solutions. Finally, we choose the remaining $N$ large crowding distance solutions as the next generation particle swarm $P_{t+1}$.

\subsection{Crowding distance sorting}

We should avoid the ordinary crowding distance sorting which is deleting the smallest crowding distance solutions. It doesn't take the crowding distance change to other solutions after each solution is deleted into account. Therefore, this paper propses a method that we eliminate the current minimum crowding distance solution at every turn. It can obtain the distribution uniformity of solutions.

\subsection{Leading particles selection}

Leading particles are including global best particles and personal best particles. For the sake of ensuring that the particles try to move closer to the true Pareto front, we choose a set of Pareto optimal front solutions randomly as the global best particles. Make the lower non-dominated front particles and the lowest non-dominated front particles have the same probability to be the global best particles[6]. Personal best particles are decided by the relationship of domination and nondomination. When the current particles dominate the personal best particles, the current particles are updated as the the personal best particles. Or do not update the personal best particles.

\subsection{Small probability random mutation}

To keep the diversity of solutions and global searching capabilities of the algorithm, this paper introduces a small probability random mutation. In the evolutionary process of PSO, we add a random mutation mechanism. It can produce a small range disturbance of particles positions to enhance the global searching ability of the algorithm[3]. Positions of the particles adopt polynomial mutation rules[7], and the mutation probability $\mathrm{Pm}$ is a small constant which belongs [0,1].

\section{Example calculation results and analysis}

\subsection{Simulation parameters setting}

In this paper, we do a environmetal and economic load dispatch simulation on IEEE30 system. The detailed parameters of IEEE30 system can refer to the literature[8]. The parameters of 6 generators are shown in Table 1. Set the number of improved PSO iterations is 100; the population of particles is 100 ; the initial inertia weight $\mathrm{w} 0=0.3$; initial learning factor $\mathrm{c} 0=0.9$; black hole radius $\mathrm{R}=8$; black hole magnetism probability $\mathrm{p}=0.3$. 
because of the Different efficiency of each generators' desulfurization and denitration equipments, we use the average values to calculate the gas emissions. It is that the absorption rate of $\mathrm{SO}_{2}$ is $98 \%$, the absorption rate of $\mathrm{NO}_{x}$ is $85 \%$ [9]. There is no absorber to absob the $\mathrm{CO}_{2}$ emissions. The consumption of 1 ton coal in power plants will release 2.62 tons $\mathrm{CO}_{2}, 8.5 \mathrm{~kg} \mathrm{SO}$ and $7.4 \mathrm{~kg} \mathrm{NO}$. Bedides, IEEE30 system only has the $\mathrm{CO}_{2}$ emission parameters. Therefore, this paper only considers the emission influence of $\mathrm{CO}_{2}$.

The emission parameters of $\mathrm{CO}_{2}$ is given in Table 2; the load needs of IEEE30 system are according to a typical summer day which is writen in Table 3. According to the power industry emission standards[10], the environmental values and punishment index of unit $\mathrm{CO}_{2}$ emission are represented in Table 4.

Table 1: Basic parameters of generators

\begin{tabular}{cccccc}
\hline Generator & $\mathrm{a}$ & $\mathrm{b}$ & $\mathrm{c}$ & $P_{G \max } / \mathrm{MW}$ & $P_{G \min } / \mathrm{MW}$ \\
\hline G1 & 0.0079 & 1.9 & 25 & 50 & 5 \\
G2 & 0.0016 & 1.9 & 40 & 60 & 5 \\
G3 & 0.0020 & 1.9 & 60 & 100 & 5 \\
G4 & 0.0011 & 2.2 & 95 & 120 & 5 \\
G5 & 0.0030 & 2.1 & 120 & 100 & 5 \\
G6 & 0.0020 & 2.0 & 75 & 60 & 5 \\
\hline
\end{tabular}

Table 2: $\mathrm{CO}_{2}$ emissions parameters

\begin{tabular}{cccccc}
\hline Generator & $\alpha_{C}$ & $\beta_{C}$ & $\gamma_{C}$ & $k_{C}$ & $\lambda_{C}$ \\
\hline G1 & 0.017 & -0.810 & 80 & $2.00 \times 10^{-6}$ & 0.0285 \\
G2 & 0.011 & -0.554 & 30 & $5.00 \times 10^{-6}$ & 0.0333 \\
G3 & 0.016 & -0.554 & 50 & $0.01 \times 10^{-6}$ & 0.0800 \\
G4 & 0.011 & -0.954 & 70 & $2.00 \times 10^{-6}$ & 0.0200 \\
G5 & 0.009 & -0.610 & 45 & $0.01 \times 10^{-6}$ & 0.0800 \\
G6 & 0.010 & -0.600 & 37 & $0.10 \times 10^{-6}$ & 0.0667 \\
\hline
\end{tabular}

Table 3: Each period load demand

\begin{tabular}{cccc}
\hline Time period & Load/MW & Time period & Load/MW \\
\hline 0 & 125.8 & 12 & 283.4 \\
1 & 118.9 & 13 & 320.8 \\
2 & 100.5 & 14 & 360.4 \\
3 & 85.6 & 15 & 358.5 \\
4 & 67.4 & 16 & 346.3 \\
5 & 76.6 & 17 & 312.6 \\
6 & 82.9 & 18 & 265.3 \\
7 & 104.1 & 19 & 242.7 \\
8 & 135.2 & 20 & 213.4 \\
9 & 168.5 & 21 & 204.1 \\
10 & 184.2 & 22 & 189.4 \\
11 & 205.2 & 23 & 165.2 \\
\hline
\end{tabular}

Table 4: Pollution emission standards of electric industry

\begin{tabular}{cc}
\hline Index & $\mathrm{CO}_{2}$ \\
\hline Environmental value & 0.002875 \\
Punishment ratio & 0.00125 \\
\hline
\end{tabular}




\subsection{Simulation results analysis}

The load of the 12-th time interval is 283.4MW. Its Simulation results can be seen in Fig.3.

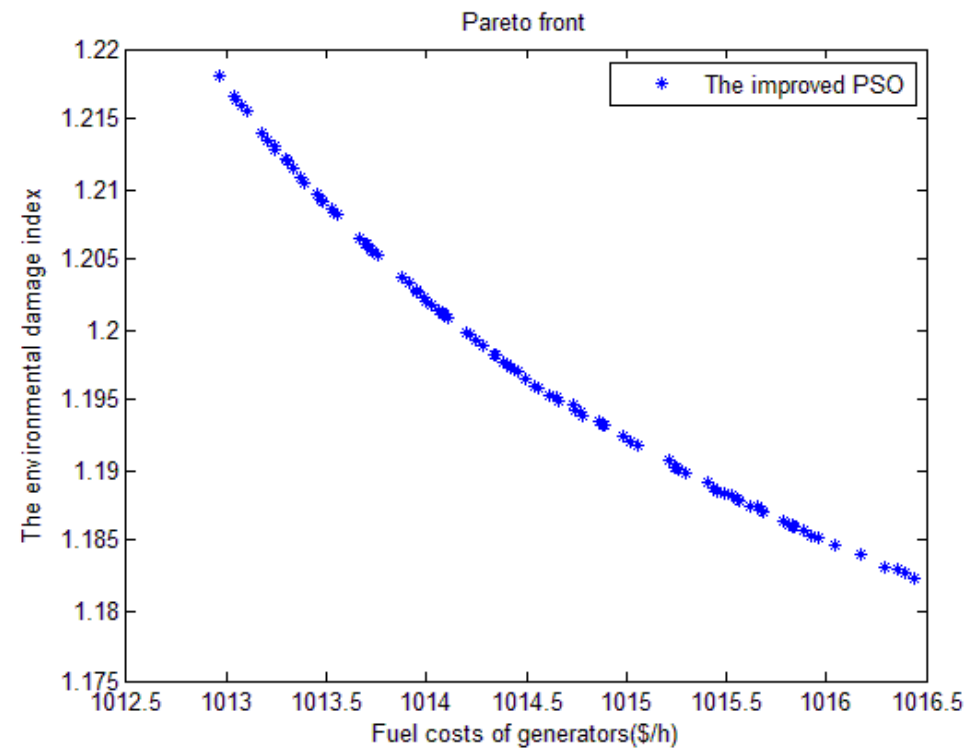

Fig.3: The optimal dispatch pareto front of 283.4MW

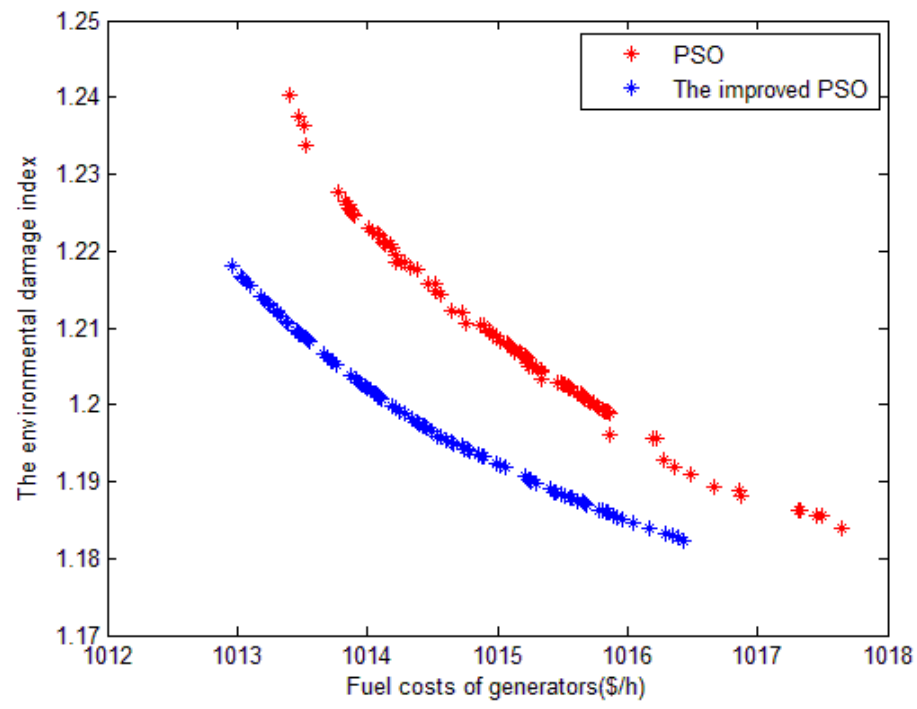

Fig.4: The pareto front comparison of algorithms

As we can see from the above Fig.3, the proposed algorithm in this paper can calculate the Pareto optimal solution set of multi-objective functions. The relationship of the two-objective functions can be illuminated directly. The number of its optimal front solutions is 100 . According to a certain preference, we can choose the appropriate optimal solutions.

Comparing standard PSO algorithm with improved PSO algorithm, and its comparison results are shown in Fig.4. It can be seen that the improved PSO has a better global searching capability which can get closer to the true Pareto front. What's more, it can obtain the diversity and distribution uniformity of solutions .

Depending on the load demands of each period, choose the compromised solutions considering the fuel costs and environmental damage index. Its each period environmental and economic load dispatch results are given as Fig.5 and Fig.6. 


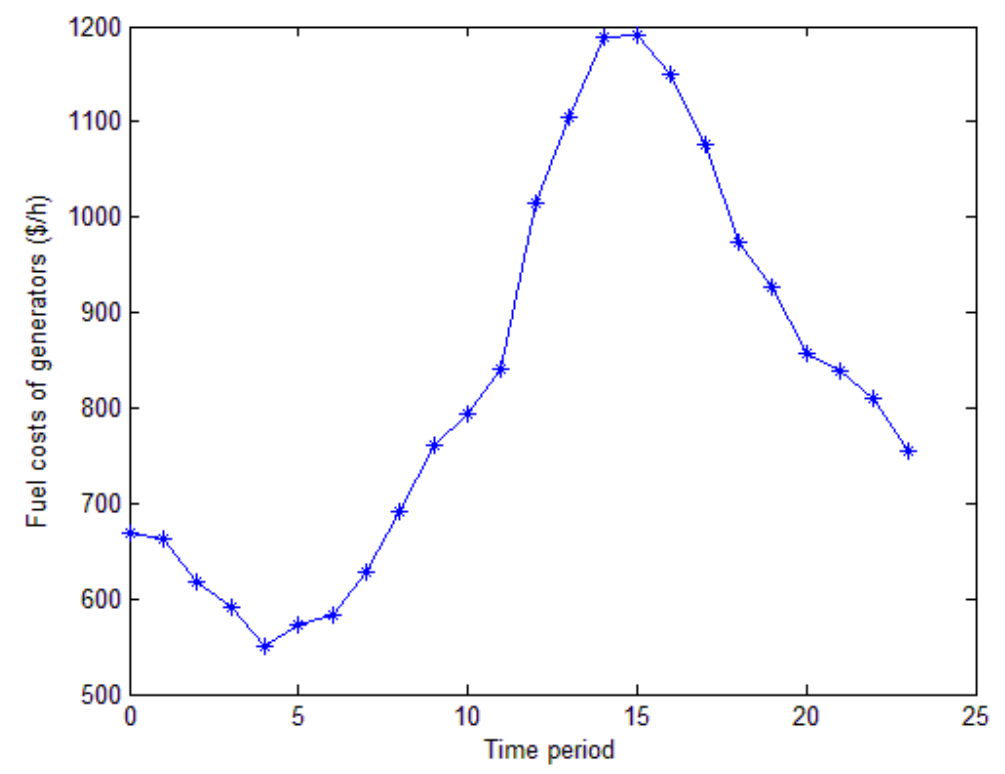

Fig.5: Fuel costs curve of each period

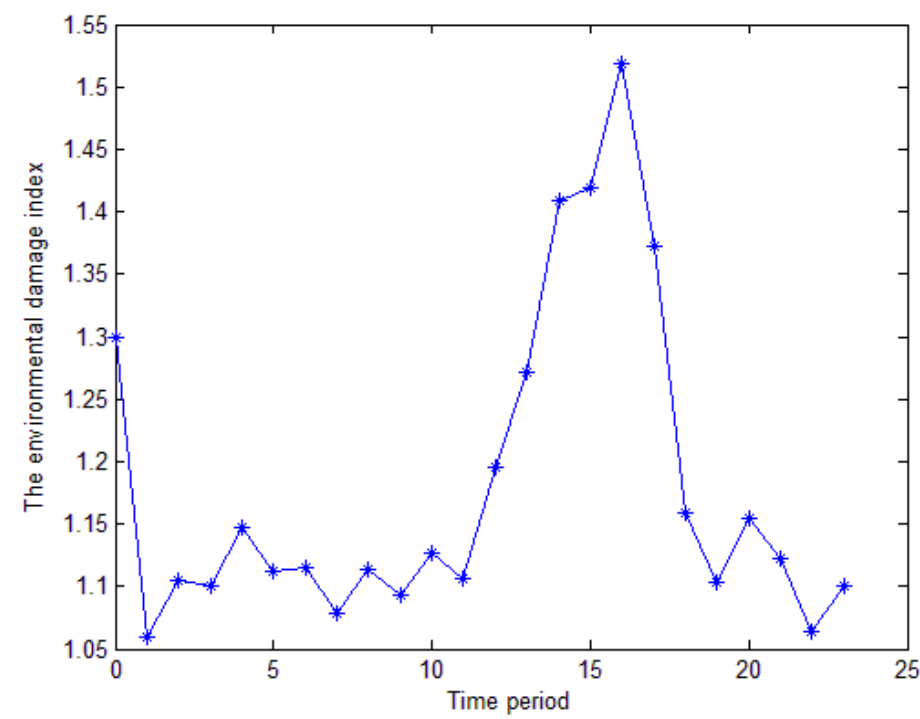

Fig.6: The environmental damage index of each period

As we can see from Fig.5 and Fig.6, the different load demands, the different fuel costs and $\mathrm{CO}_{2}$ environmental damage index. Fig.7 is the output active power optimal dispatch of generators each period. 


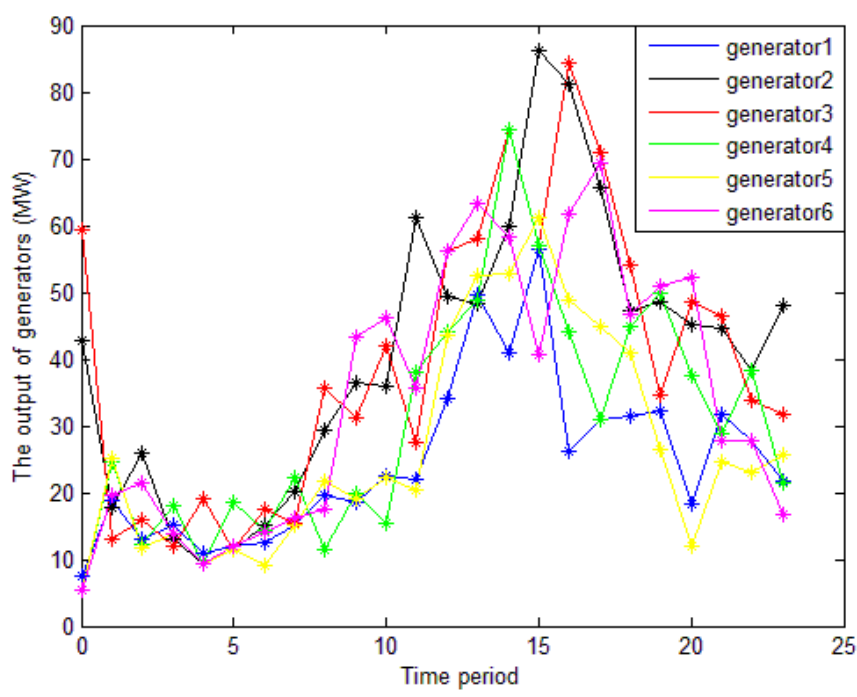

Fig.7: The generators output of each period

\section{Summary}

For the defect of the standard PSO algorithm, this paper presents an improved particle swarm optimization algorithm. We apply it to search the Pareto optimal front solution sets. For specific, we use it to solve the environmental and economic load dispatch problem of power systems and get the satisfied results. The simulation results show that the proposed algorithm can search Pareto front effectively, and the solutions have the diversity and uniformity. Considering the environmental impact of $\mathrm{CO}_{2}$ emissions and the minimum fuel consumption costs of generators, we get the optimal dispatch of generators output depending on the every period load needs.

\section{References}

[1] Qian Ke-jun, Yuan yue, Shi Xiao-dan, etc. Environmental benefits analysis of distributed generation. Proceedings of the CSEE, 28(29), pp. 11-15,2008.

[2] Zhou Ren-jun, Ran Xiao-hong, Mao Fa-long, etc. Energy-saving coordinated optimal disptch of distributed combined cool, heat and power supply. Power System Technolgy, 36(6), pp. 8$14,2012$.

[3] Li Zhong-kai, Tan Jian-rong, Feng Yi-xiong, etc. Multi-objective particle swarm optimization algorithm based on crowding distance sorting and its application. Computer Integrated Manufacturing Systems, 14(7), pp. 1329-1336,2008.

[4] Zhang Jun-qi, Liu Kun, etc. Random black hole particle swarm optimization and its application. Proceedings of IEEE Conference on Neural \& Signal Processing. Zhenjiang(China)2008: 359365.

[5] Cheng Shan. The multi-objective optimization of the distributed generators on the Distribution network. Chongqin:Chongqi University,2013.

[6] Chen Min-you, Zhang Cong-yu, Luo Ci-yong. Adaptive evolutionary multi-objective particle swarm optimization algorithm. Control and Decision, 24(12), pp. 1851-1864,2009.

[7] Debk, Jains. Multi-speed gearbox design using multi-objective evolutionary algorithms. Journal of Mechanical Design, 125(3), pp. 609-619,2003.

[8] ABIDO M A. Multi-objective particle swarm optimization for environmental / economic dispatch problem. Electric Power Systems Research, 79(7), pp. 1105-1113,2003.

[9] Zhao Jian-wei. Economicemission load dispatch in power systems based on improved particle swarm optimization algorithm. Shandong:Qing dao University,2002. 
[10] Zhou Ren-jun, Li Shao-jin, etc. Space particle swarm optimization algorithm and its application in environmental \& economic load distribution of power system. Electric Power Automation Equipment, 34(9), pp. 7-12,2014. 